\title{
Some Inequalities for Power Series of Two Operators in Hilbert Spaces
}

\author{
Sever Silvestru DRAGOMIR and Mitsuru UCHIYAMA
}

Victoria University and Shimane University

(Communicated by K. Takemura)

\begin{abstract}
Some inequalities for functions defined by power series concerning two operators in both the noncommutative and commutative case are given. Natural examples for fundamental functions that can be represented by power series are presented as well.
\end{abstract}

\section{Introduction}

For power series $f(z)=\sum_{n=0}^{\infty} a_{n} z^{n}$ with complex coefficients we can naturally construct another power series which have as coefficients the absolute values of the coefficient of the original series, namely, $f_{a}(z):=\sum_{n=0}^{\infty}\left|a_{n}\right| z^{n}$. It is obvious that this new power series have the same radius of convergence as the original series, and that if all coefficients $a_{n} \geq 0$, then $f_{a}=f$.

With this notation S.S. Dragomir [4] (also see [5]) showed the following:

Let $f(z)=\sum_{n=0}^{\infty} a_{n} z^{n}$ be a function defined by power series with complex coefficients and convergent on the open disk $D(0, R) \subset C, R>0$. Let $T \in B(H), \alpha, \beta \geq 0$ with $\alpha+\beta \geq 1$ and such that

$$
\|T\|^{2 \alpha},\|T\|^{2 \beta}<R .
$$

Then

$$
\begin{aligned}
& \left|\left\langle T f\left(|T|^{\alpha+\beta}\right)|T|^{\alpha+\beta-1} x, y\right\rangle\right|^{2} \\
& \leq\left\langle f_{a}\left(|T|^{2 \alpha}\right)|T|^{2 \alpha} x, x\right\rangle\left\langle f_{a}\left(\left|T^{*}\right|^{2 \beta}\right)\left|T^{*}\right|^{2 \beta} y, y\right\rangle
\end{aligned}
$$

for any $x, y \in H$.

This is an extension of the following inequality for a bounded linear operator $T \in B(H)$ by Furuta [7] (also see [8]):

$$
\left|\left\langle T|T|^{\alpha+\beta-1} x, y\right\rangle\right|^{2} \leq\left\langle|T|^{2 \alpha} x, x\right\rangle\left\langle\left|T^{*}\right|^{2 \beta} y, y\right\rangle, \quad x, y \in H .
$$

Received August 8, 2012; revised November 19, 2012 1991 Mathematics Subject Classification: 47A63; 47A99

Key words and phrases: Bounded linear operators, Functions of operators, Normal operators, Power series 
Motivated by this result for one operator, we investigate in the current paper some inequalities for functions defined by power series concerning two operators in both the noncommutative and commutative case. In particular, for $p>1, \frac{1}{p}+\frac{1}{q}=1$ we show that

$$
\|f(A B)\| \leq f_{a}^{1 / p}\left(\|A\|^{p}\right) f_{a}^{1 / q}\left(\|B\|^{q}\right) .
$$

Moreover we prove this inequality is also valid for every unitarily invariant norm.

The following is one among some examples given in this paper:

If $\|A\|^{p},\|B\|^{q}<1$, then

$$
\left\|\left(1_{H} \pm A B\right)^{-1}\right\| \leq\left(1-\|A\|^{p}\right)^{-1 / p}\left(1-\|B\|^{q}\right)^{-1 / q} .
$$

\section{Some General Norm Inequalities}

The following result concerning norm inequalities for two bounded operators may be stated:

THEOREM 1. Let $f(z)=\sum_{n=0}^{\infty} a_{n} z^{n}(\neq 0)$ be a function defined by power series with complex coefficients and convergent on the open disk $D(0, R) \subset \mathbf{C}, R>0$. If $A$ and $B$ are two bounded operators on the Hilbert space $H$ and for $p>1, \frac{1}{p}+\frac{1}{q}=1$

$$
\|A\|^{p},\|B\|^{q}<R,
$$

then

$$
\|f(A B)\| \leq \min \left\{K_{1}(p, q), K_{2}(p, q)\right\}
$$

where

$$
K_{1}(p, q):=f_{a}^{1 / p}\left(\|A\|^{p}\right) f_{a}^{1 / q}\left(\|B\|^{q}\right)
$$

and

$$
K_{2}(p, q):=\frac{f_{a}\left(\|A\|^{p}\right) f_{a}\left(\|B\|^{q}\right)}{f_{a}\left(\|A\|^{p-1}\|B\|^{q-1}\right)} .
$$

Proof. By the properties of operator norm, observe that, for any $j \in \mathbf{N}$ we have

$$
\left\|(A B)^{j}\right\| \leq\|A\|^{j}\|B\|^{j} .
$$

If we multiply with $\left|a_{j}\right|$ and use the generalized triangle inequality we have

$$
\left\|\sum_{j=0}^{n} a_{j}(A B)^{j}\right\| \leq \sum_{j=0}^{n}\left|a_{j}\right|\|A\|^{j}\|B\|^{j}
$$

for any $n \in \mathbf{N}$. 
Now, by Hölder's inequality we have

$$
\sum_{j=0}^{n}\left|a_{j}\right|\|A\|^{j}\|B\|^{j} \leq\left(\sum_{j=0}^{n}\left|a_{j}\right|\|A\|^{j p}\right)^{1 / p}\left(\sum_{j=0}^{n}\left|a_{j}\right|\|B\|^{j q}\right)^{1 / q}
$$

for any $n \in \mathbf{N}$, and by (2.5) we get

$$
\left\|\sum_{j=0}^{n} a_{j}(A B)^{j}\right\| \leq\left(\sum_{j=0}^{n}\left|a_{j}\right|\|A\|^{j p}\right)^{1 / p}\left(\sum_{j=0}^{n}\left|a_{j}\right|\|B\|^{j q}\right)^{1 / q} .
$$

Since the series whose partial sums are involved in (2.7) are convergent, then by taking $n \rightarrow \infty$ in (2.7) we deduce the first inequality in (2.3).

Further, by utilizing the following Hölder's type inequality obtained by Dragomir and Sándor in 1990 [6] (see also [2, Corollary 2.34]):

$$
\sum_{k=0}^{n} m_{k}\left|x_{k}\right|^{p} \sum_{k=0}^{n} m_{k}\left|y_{k}\right|^{q} \geq \sum_{k=0}^{n} m_{k}\left|x_{k} y_{k}\right| \sum_{k=0}^{n} m_{k}\left|x_{k}\right|^{p-1}\left|y_{k}\right|^{q-1}
$$

that holds for nonnegative numbers $m_{k}$ and complex numbers $x_{k}, y_{k}$ where $k \in\{0, \ldots, n\}$, we observe that the convergence of the series $\sum_{k=0}^{\infty} m_{k}\left|x_{k}\right|^{p}$ and $\sum_{k=0}^{\infty} m_{k}\left|y_{k}\right|^{q}$ imply the convergence of the series $\sum_{k=0}^{\infty} m_{k}\left|x_{k}\right|^{p-1}\left|y_{k}\right|^{q-1}$.

Utilising (2.8) we then have

$$
\sum_{j=0}^{n}\left|a_{j}\right|\|A\|^{j}\|B\|^{j} \leq \frac{\sum_{j=0}^{n}\left|a_{j}\right|\|A\|^{j p} \sum_{j=0}^{n}\left|a_{j}\right|\|B\|^{j q}}{\sum_{j=0}^{n}\left|a_{j}\right|\|A\|^{j(p-1)}\|B\|^{j(q-1)}}
$$

which together with (2.5) gives

$$
\left\|\sum_{j=0}^{n} a_{j}(A B)^{j}\right\| \leq \frac{\sum_{j=0}^{n}\left|a_{j}\right|\|A\|^{j p} \sum_{j=0}^{n}\left|a_{j}\right|\|B\|^{j p}}{\sum_{j=0}^{n}\left|a_{j}\right|\|A\|^{j(p-1)}\|B\|^{j(q-1)}}
$$

for any $n \in \mathbf{N}$.

Since all the series whose partial sums are involved in (2.9) are convergent, then by taking $n \rightarrow \infty$ in (2.9) we deduce the second inequality in (2.2).

REMARK 1. The case $p=q=2$ produces the Schwarz's type inequality

$$
\|f(A B)\|^{2} \leq f_{a}\left(\|A\|^{2}\right) f_{a}\left(\|B\|^{2}\right),
$$

provided $\|A\|^{2},\|B\|^{2}<R$.

The finite-dimensional case is as follows: 
THEOREM 2. Theorem 1 also holds for every unitarily invariant norm $\||\cdot|\|$ on a finite matrix algebra. Moreover, we have the inequalities

$$
\|f(A B)\| \leq \min \left\{L_{1}(p, q), L_{2}(p, q)\right\}
$$

where

$$
L_{1}(p, q):=f_{a}^{1 / p}\left(\left\|\left.|| A\right|^{p}\right\| \mid\right) f_{a}^{1 / q}\left(\left\|\left.|| B\right|^{q}\right\|\right)
$$

and

$$
L_{2}(p, q):=\frac{f_{a}\left(\left\||||A|^{p}\right\|||\right) f_{a}\left(\left\|\left.|| B\right|^{q}\right\|\right)}{f_{a}\left(\left\|\left.|| A\right|^{p}\left|\left\|^{1 / q}\right\|\right||B|^{q}\right\|^{1 / p}\right)},
$$

provided $\left\|\left.|| A\right|^{p}\right\|\left|,\left\||B|^{q}\right\|\right|<R$.

Proof. Since $\|A B\| \leq\|A\|\|\cdot\| B \|$ and $\|A B\| \leq\left.\|\||A|^{p}\left\|^{1 / p} \cdot\right\||| B\right|^{q} \|^{1 / q}$ where $p>1, \frac{1}{p}+\frac{1}{q}=1$ (see for instance [1, p. 95]), we have by the Hölder inequality that

$$
\begin{aligned}
\left\|\sum_{j=0}^{n} a_{j}(A B)^{j} \mid\right\| & \leq\left.\left.\sum_{j=0}^{n}\left|a_{j}\right|\left\|\left|A B\left\|^{j} \leq \sum_{j=0}^{n}\left|a_{j}\right|\right\|\right||A|^{p}\right\|\right|^{j / p} \cdot\left\|\left.|| B\right|^{q}\right\|\right|^{j / q} \\
& \leq\left(\sum_{j=0}^{n}\left|a_{j}\left\||A|^{p}\right\|^{j}\right)^{1 / p}\left(\sum_{j=0}^{n}\left|a_{j}\right|\left\|\left.|| B\right|^{q}\right\|^{j}\right)^{1 / q}\right.
\end{aligned}
$$

for any $n \in \mathbf{N}$.

Since all the series whose partial sums are involved in (2.14) are convergent, then by taking $n \rightarrow \infty$ in (2.14) we deduce the first inequality in (2.11).

Utilising the inequality (2.8) we also have

$$
\begin{aligned}
& \sum_{j=0}^{n}\left|a_{j}\right|\left\|\left.|| A\right|^{p}\right\|^{j / p} \cdot \|\left.|| B\right|^{q}||^{j / q} \\
& \leq \frac{\sum_{j=0}^{n}\left|a_{j}\right|\left|\left\||A|^{p}\right\|^{j} \sum_{j=0}^{n}\right| a_{j}\left|\left\|\left.|| B\right|^{q}\right\|\right|^{j}}{\sum_{j=0}^{n}\left|a_{j}\right|\left|\left\||A|^{p}\right\|^{j \frac{p-1}{p}} \cdot\left\|\left.|| B\right|^{q}\right\|^{j \frac{q-1}{q}}\right.} \\
& \quad=\frac{\sum_{j=0}^{n}\left|a_{j}\right|\left\|\left.|| A\right|^{p}\right\|^{j} \sum_{j=0}^{n}\left|a_{j}\right|\left\|\left.|| B\right|^{q}\right\|^{j}}{\sum_{j=0}^{n}\left|a_{j}\right|\left\|\left.|| A\right|^{p}\right\|^{\frac{j}{q}} \cdot\left\|\left.|| B\right|^{q}\right\|^{\frac{j}{p}}}
\end{aligned}
$$

for any $n \in \mathbf{N}$.

Since all the series whose partial sums are involved in (2.15) are convergent, then by taking $n \rightarrow \infty$ in (2.15) we deduce the first inequality in (2.11).

REMARK 2. The case $p=q=2$ produces the Schwarz's type inequality

$$
\|f(A B)\|^{2} \leq f_{a}\left(\left\|\left.|| A\right|^{2}\right\| \mid\right) f_{a}\left(\left\|\left.|| B\right|^{2}\right\| \mid\right),
$$


provided $\left\||A|^{2}\right\|||,\left\||B|^{2}\right\| \mid<R$.

A refinement of the inequality (2.10) may be found in the following theorem:

THEOREM 3. Let $f(z)=\sum_{n=0}^{\infty} a_{n} z^{n}(\neq 0)$ be a function defined by power series with complex coefficients and convergent on the open disk $D(0, R) \subset \mathbf{C}, R>0$. If $A$ and $B$ are two bounded operators on the Hilbert space $H$ and

$$
\|A\|^{2},\|B\|^{2}<R
$$

then

$$
\begin{aligned}
\|f(A B)\|^{2} & \leq f_{a}\left(\|A\|^{1+\alpha}\|B\|^{1-\alpha}\right) f_{a}\left(\|A\|^{1-\alpha}\|B\|^{1+\alpha}\right) \\
& \leq f_{a}\left(\|A\|^{2}\right) f_{a}\left(\|B\|^{2}\right)
\end{aligned}
$$

where $\alpha \in[0,1]$.

If $\sum_{n=0}^{\infty}\left|a_{n}\right|<\infty$ and in addition to the condition (2.16) we have $\|A\|,\|B\|<R$, then

$$
\|f(A B)\| \leq f_{a}(1) \cdot \frac{f_{a}\left(\|A\|^{2}\right) f_{a}\left(\|B\|^{2}\right)}{f_{a}(\|A\|) f_{a}(\|B\|)} .
$$

ProOF. We utilize the Callebaut inequality (see for instance [2, Remark 3.31])

$$
\left(\sum_{j=1}^{n} p_{j} a_{j} b_{j}\right)^{2} \leq \sum_{j=1}^{n} p_{j} a_{j}^{1+\alpha} b_{j}^{1-\alpha} \sum_{j=1}^{n} p_{j} a_{j}^{1-\alpha} b_{j}^{1+\alpha} \leq \sum_{j=1}^{n} p_{j} a_{j}^{2} \sum_{j=1}^{n} p_{j} b_{j}^{2}
$$

that holds for $\alpha \in[0,1]$ and the nonnegative numbers $a_{j}, b_{j}, p_{j}$ with $j \in\{1, \ldots, n\}$.

Therefore

$$
\begin{aligned}
\left(\sum_{j=0}^{n}\left|a_{j}\right|\|A\|^{j}\|B\|^{j}\right)^{2} & \leq \sum_{j=0}^{n}\left|a_{j}\right|\|A\|^{(1+\alpha) j}\|B\|^{(1-\alpha) j} \sum_{j=0}^{n}\left|a_{j}\right|\|A\|^{(1-\alpha) j}\|B\|^{(1+\alpha) j} \\
& \leq \sum_{j=0}^{n}\left|a_{j}\right|\|A\|^{2 j} \sum_{j=0}^{n}\left|a_{j}\right|\|B\|^{2 j}
\end{aligned}
$$

and by (2.5) we get

$$
\begin{aligned}
& \left\|\sum_{j=0}^{n} a_{j}(A B)^{j}\right\|^{2} \\
& \quad \leq \sum_{j=0}^{n}\left|a_{j}\right|\|A\|^{(1+\alpha) j}\|B\|^{(1-\alpha) j} \sum_{j=0}^{n}\left|a_{j}\right|\|A\|^{(1-\alpha) j}\|B\|^{(1+\alpha) j} \\
& \quad \leq \sum_{j=0}^{n}\left|a_{j}\right|\|A\|^{2 j} \sum_{j=0}^{n}\left|a_{j}\right|\|B\|^{2 j}
\end{aligned}
$$


for any $n \in \mathbf{N}$.

Since all the series whose partial sums are involved in (2.19) are convergent, then by taking $n \rightarrow \infty$ in (2.19) we deduce the inequality (2.17).

For the second part, we use the following inequality obtained by S.S. Dragomir in 1984 [3] (see also [2, Theorem 2.20]):

$$
\frac{\sum_{j=1}^{n} p_{j} a_{j} b_{j} \sum_{j=1}^{n} p_{j} a_{j} \sum_{j=1}^{n} p_{j} b_{j}}{\sum_{j=1}^{n} p_{j}} \leq \sum_{j=1}^{n} p_{j} a_{j}^{2} \sum_{j=1}^{n} p_{j} b_{j}^{2}
$$

that holds for the nonnegative numbers $a_{j}, b_{j}, p_{j}$ with $j \in\{1, \ldots, n\}$ and $\sum_{j=1}^{n} p_{j}>0$.

Utilising this inequality, we have

$$
\sum_{j=0}^{n}\left|a_{j}\right|\|A\|^{j}\|B\|^{j} \leq \sum_{j=0}^{n}\left|a_{j}\right| \cdot \frac{\sum_{j=0}^{n}\left|a_{j}\right|\|A\|^{2 j} \sum_{j=0}^{n}\left|a_{j}\right|\|B\|^{2 j}}{\sum_{j=0}^{n}\left|a_{j}\right|\|A\|^{2} \sum_{j=0}^{n}\left|a_{j}\right|\|B\|^{2}}
$$

which together with $(2.5)$ produces

$$
\left\|\sum_{j=0}^{n} a_{j}(A B)^{j}\right\| \leq \sum_{j=0}^{n}\left|a_{j}\right| \cdot \frac{\sum_{j=0}^{n}\left|a_{j}\right|\|A\|^{2 j} \sum_{j=0}^{n}\left|a_{j}\right|\|B\|^{2 j}}{\sum_{j=0}^{n}\left|a_{j}\right|\|A\|^{2} \sum_{j=0}^{n}\left|a_{j}\right|\|B\|^{2}} .
$$

Since all the series whose partial sums are involved in (2.20) are convergent, then by taking $n \rightarrow \infty$ in (2.20) we deduce the inequality (2.18).

REMARK 3. The condition $f_{a}(1)<\infty$ can be avoided if a complex parameter $|z|<R$ is introduced. Namely, we can obtain the following generalization of (2.18)

$$
\|f(z A B)\| \leq f_{a}(|z|) \cdot \frac{f_{a}\left(|z|\|A\|^{2}\right) f_{a}\left(|z|\|B\|^{2}\right)}{f_{a}(|z|\|A\|) f_{a}(|z|\|B\|)},
$$

provided $|z|\|A\|^{2},|z|\|B\|^{2},|z|\|A\|,|z|\|B\|<R$.

The finite-dimensional version of Theorem 3 is as follows:

THEOREM 4. Theorem 3 also holds for every unitarily invariant norm $|\| \cdot|||$ on a finite matrix algebra. Moreover, we have the inequalities

$$
\begin{aligned}
& \|f(A B)\|^{2} \\
& \quad \leq f_{a}\left(\left.\left.\left\||A|^{2}\right\|\right|^{\frac{1+\alpha}{2}}\left\||B|^{2}\right\|\right|^{\frac{1-\alpha}{2}}\right) f_{a}\left(\left.\left\||A|^{2}\right\|\right|^{\frac{1-\alpha}{2}}\left\||B|^{2}\right\| \|^{\frac{1+\alpha}{2}}\right) \\
& \quad \leq f_{a}\left(\left\||A|^{2}\right\|\right) f_{a}\left(\left\||B|^{2}\right\|\right),
\end{aligned}
$$

provided

$$
\left.\left\|\left.|| A\right|^{2}\right\||,||| B\right|^{2} \| \mid<R,
$$

where $\alpha \in[0,1]$. 
If $\sum_{n=0}^{\infty}\left|a_{n}\right|<\infty$ and in addition to the condition (2.23) we have

$$
\left.\left\||A|^{2}\right\|\right|^{1 / 2},\left.\left\||B|^{2}\right\|\right|^{1 / 2}<R
$$

then

$$
\|f(A B)\| \leq f_{a}(1) \cdot \frac{f_{a}\left(\left\|\left.|| A\right|^{2}\right\| \mid\right) f_{a}\left(\|\left.|| B\right|^{2}||\right)}{f_{a}\left(\left.\left\|\left.|| A\right|^{2}\right\|\right|^{1 / 2}\right) f_{a}\left(\|\left.|| B\right|^{2}||^{1 / 2}\right)} .
$$

The details of the proof are left to the reader.

\section{Some Vector Inequalities for Normal Operators}

The case of normal operators is as follows:

THEOREM 5. Let $f(z)=\sum_{n=0}^{\infty} a_{n} z^{n}$ be a function defined by power series with complex coefficients and convergent on the open disk $D(0, R) \subset \mathbf{C}, R>0$. If $A$ and $B$ are two commuting normal operators on the Hilbert space $H, z \in \mathbf{C}$ and

$$
|z|\|A\|^{2},|z|\|B\|^{2}<R
$$

then we have

$$
|\langle f(z A B) x, y\rangle|^{2} \leq\left\langle f_{a}\left(|z||A|^{2}\right) x, x\right\rangle\left\langle f_{a}\left(|z||B|^{2}\right) y, y\right\rangle
$$

for any $x, y \in H$.

Proof. By utilizing Schwarz inequality we have for any $x, y \in H$ that

$$
\left|\left\langle A^{j} x,\left(B^{*}\right)^{j} y\right\rangle\right|^{2} \leq\left\langle A^{j} x, A^{j} x\right\rangle\left\langle\left(B^{*}\right)^{j} y,\left(B^{*}\right)^{j} y\right\rangle
$$

for any $j \in \mathbf{N}$, which in operator modulus notations is equivalent with

$$
\left|\left\langle B^{j} A^{j} x, y\right\rangle\right|^{2} \leq\left\langle\left|A^{j}\right|^{2} x, x\right\rangle\left\langle\left|\left(B^{*}\right)^{j}\right|^{2} y, y\right\rangle .
$$

Since $A$ and $B$ are normal operators, then

$$
\left|A^{j}\right|^{2}=|A|^{2 j} \quad \text { and } \quad\left|\left(B^{*}\right)^{j}\right|^{2}=|B|^{2 j}
$$

for any $j \in \mathbf{N}$.

By the commutativity of $A$ with $B$ we also have

$$
B^{j} A^{j}=(A B)^{j}
$$

for any $j \in \mathbf{N}$ and then by (3.3) we have

$$
\left|\left\langle(A B)^{j} x, y\right\rangle\right|^{2} \leq\left\langle|A|^{2 j} x, x\right\rangle\left\langle|B|^{2 j} y, y\right\rangle
$$

for any $x, y \in H$ and for any $j \in \mathbf{N}$. 
If we multiply the inequality (3.3) with $\left|a_{j}\right||z|^{j}$, sum over $j$ from 0 to $m$ and use the generalized triangle inequality and the Cauchy-Bunyakovsky-Schwarz weighted inequality, we have successively

$$
\begin{aligned}
& \left|\left\langle\sum_{j=0}^{m} a_{j} z^{j}(A B)^{j} x, y\right\rangle\right| \\
& \quad \leq \sum_{j=0}^{m}\left|a_{j}\right||z|^{j}\left|\left\langle(A B)^{j} x, y\right\rangle\right| \\
& \quad \leq \sum_{j=0}^{m}\left|a_{j}\right||z|^{j}\left\langle|A|^{2 j} x, x\right\rangle^{1 / 2}\left\langle|B|^{2 j} y,\left.y\right|^{1 / 2}\right. \\
& \quad \leq\left(\sum_{j=0}^{m}\left|a_{j}\right||z|^{j}\left\langle|A|^{2 j} x, x\right\rangle\right)^{1 / 2}\left(\sum_{j=0}^{m}\left|a_{j}\right||z|^{j}\left\langle|B|^{2 j} y, y\right\rangle\right)^{1 / 2} \\
& \quad=\left\langle\sum_{j=0}^{m}\left|a_{j}\right||z|^{j}|A|^{2 j} x, x\right\rangle^{1 / 2}\left\langle\sum_{j=0}^{m}\left|a_{j}\right||z|^{j}|B|^{2 j} y, y\right\rangle^{1 / 2}
\end{aligned}
$$

for any $x, y \in H$ and for any $m \in \mathbf{N}$.

Since the series $\sum_{j=0}^{\infty}\left|a_{j}\right||z|^{j}|A|^{2 j}, \sum_{j=0}^{\infty}\left|a_{j}\right||z|^{j}|B|^{2 j}$ and $\sum_{j=0}^{\infty} a_{j} z^{j}(A B)^{j}$ are convergent, then by taking the limit over $m \rightarrow \infty$ in (3.5) we deduce the desired result (3.2).

COROLLARY 1. Let $f(z)=\sum_{n=0}^{\infty} a_{n} z^{n}$ be a function defined by power series with real coefficients and convergent on the open disk $D(0, R) \subset \mathbf{C}, R>0$. If $A$ and $B$ are two commuting normal operators on the Hilbert space $H$ satisfying the condition (3.1) then we have the norm inequality

$$
\|f(z A B)\|^{2} \leq\left\|f_{a}\left(|z||A|^{2}\right)\right\|\left\|f_{a}\left(|z||B|^{2}\right)\right\|
$$

and the numerical radius inequality

$$
w[f(z A B)] \leq \frac{1}{2}\left\|f_{a}\left(|z||A|^{2}\right)+f_{a}\left(|z||B|^{2}\right)\right\| .
$$

Proof. From (3.2) we also have the inequalities

$$
\begin{aligned}
|\langle f(z A B) x, x\rangle| & \leq\left\langle f_{a}\left(|z||A|^{2}\right) x, x\right\rangle^{1 / 2}\left\langle f_{a}\left(|z||B|^{2}\right) x, x\right\rangle^{1 / 2} \\
& \leq \frac{1}{2}\left\langle\left[f_{a}\left(|z||A|^{2}\right)+f_{a}\left(|z||B|^{2}\right)\right] x, x\right\rangle
\end{aligned}
$$

for any $x \in H$, which, by taking the supremum over $\|x\|=1$, produces the desired result (3.7). 
REMARK 4. If $A$ is a normal operator and $z \in \mathbf{C}$ with $|z|\|A\|^{2},|z|<R$, then by taking $B=1_{H}$ in (3.2) we get

$$
|\langle f(z A) x, y\rangle|^{2} \leq f_{a}(|z|)\left\langle f_{a}\left(|z||A|^{2}\right) x, x\right\rangle\|y\|^{2}
$$

for any $x, y \in H$.

If $A$ is a normal operator and $z \in \mathbf{C}$ with $|z|\|A\|^{2},|z|<R$, then by taking $B=A$ in (3.2) we get

$$
\left|\left\langle f\left(z A^{2}\right) x, y\right\rangle\right|^{2} \leq\left\langle f_{a}\left(|z||A|^{2}\right) x, x\right\rangle\left\langle f_{a}\left(|z||A|^{2}\right) y, y\right\rangle
$$

and by taking $B=A^{*}$ in (3.2) we also get

$$
\left|\left\langle f\left(z|A|^{2}\right) x, y\right\rangle\right|^{2} \leq\left\langle f_{a}\left(|z||A|^{2}\right) x, x\right\rangle\left\langle f_{a}\left(|z||A|^{2}\right) y, y\right\rangle
$$

for any $x, y \in H$.

Moreover, if $U$ and $V$ are two commuting unitary operators, then by taking $A=U$ and $B=V$ in (3.2) we get

$$
|\langle f(z U V) x, y\rangle| \leq f_{a}(|z|)\|x\|\|y\|
$$

for any $x, y \in H$ and $z \in \mathbf{C}$ with $|z|<R$.

The following result for two power series can be stated as well:

THEOREM 6. Let $f(z)=\sum_{n=0}^{\infty} a_{n} z^{n}$ and be $g(z)=\sum_{n=0}^{\infty} b_{n} z^{n}$ be two functions defined by power series with complex coefficients and both of them convergent on the open disk $D(0, R) \subset \mathbf{C}, R>0$. If $A$ and $B$ are two normal operators on the Hilbert space $H$, $z, u \in \mathbf{C}$ and

$$
|z|\|A\|,|u|\|B\| \leq R
$$

then we have

$$
\begin{aligned}
& |\langle f(z A) x, g(u B) y\rangle|^{2} \\
& \quad \leq f_{a}\left(|z|^{2}\right) g_{a}\left(|u|^{2}\right)\left\langle f_{a}\left(|A|^{2}\right) x, x\right\rangle\left\langle g_{a}\left(|B|^{2}\right) y, y\right\rangle
\end{aligned}
$$

for any $x, y \in H$.

Proof. By Schwarz's inequality we also have the following inequality for normal operators

$$
\left|\left\langle A^{j} x, B^{k} y\right\rangle\right| \leq\left\langle|A|^{2 j} x, x\right\rangle^{1 / 2}\left\langle|B|^{2 k} y,\left.y\right|^{1 / 2}\right.
$$

for any $x, y \in H$ and $j, k \in \mathbf{N}$. 
If we multiply (3.14) with $\left|a_{j}\right||z|^{j}\left|b_{k}\right||u|^{k}$, sum over $j$ and $k$ from 0 to $m$ and use the generalized triangle inequality, then we have successively

$$
\begin{aligned}
& \left|\left\langle\sum_{j=0}^{m} a_{j} z^{j} A^{j} x, \sum_{k=0}^{m} b_{k} u^{k} B^{k} y\right\rangle\right| \\
& \quad \leq \sum_{j=0}^{m} \sum_{k=0}^{m}\left|a_{j}\right||z|^{j}\left|b_{k}\right||u|^{k}\left|\left\langle A^{j} x, B^{k} y\right\rangle\right| \\
& \quad \leq \sum_{j=0}^{m} \sum_{k=0}^{m}\left|a_{j}\right||z|^{j}\left|b_{k}\right||u|^{k}\left\langle|A|^{2 j} x, x\right\rangle^{1 / 2}\left\langle|B|^{2 k} y, y\right\rangle^{1 / 2} \\
& \quad=\sum_{j=0}^{m}\left|a_{j}\right||z|^{j}\left\langle|A|^{2 j} x, x\right\rangle^{1 / 2} \sum_{k=0}^{m}\left|b_{k}\right||u|^{k}\left\langle|B|^{2 k} y,\left.y\right|^{1 / 2}\right.
\end{aligned}
$$

for any $x, y \in H$ and $m \in \mathbf{N}$.

Further, by the Cauchy-Bunyakovsky-Schwarz inequality we also have

$$
\sum_{j=0}^{m}\left|a_{j}\right||z|^{j}\left\langle|A|^{2 j} x,\left.x\right|^{1 / 2} \leq\left(\sum_{j=0}^{m}\left|a_{j}\right||z|^{2 j}\right)^{1 / 2}\left\langle\sum_{j=0}^{m}\left|a_{j}\right||A|^{2 j} x, x\right\rangle^{1 / 2}\right.
$$

and

$$
\sum_{k=0}^{m}\left|b_{k}\right||u|^{k}\left\langle|B|^{2 k} y,\left.y\right|^{1 / 2} \leq\left(\sum_{k=0}^{m}\left|b_{k}\right||u|^{2 k}\right)^{1 / 2}\left\langle\sum_{k=0}^{m}\left|b_{k}\right||B|^{2 k} y, y\right\rangle^{1 / 2}\right.
$$

for any $x, y \in H$ and $m \in \mathbf{N}$, which together with (3.15) provide

$$
\begin{aligned}
& \mid\left\langle\sum_{j=0}^{m} a_{j} z^{j} A^{j} x, \sum_{k=0}^{m} b_{k} u^{k} B^{k} y\right\rangle \\
& \leq\left(\sum_{j=0}^{m}\left|a_{j}\right||z|^{2 j}\right)^{1 / 2}\left\langle\sum_{j=0}^{m}\left|a_{j}\right||z|^{j}|A|^{2 j} x, x\right\rangle^{1 / 2} \\
& \times\left(\sum_{k=0}^{m}\left|b_{k}\right||u|^{2 k}\right)^{1 / 2}\left\langle\sum_{k=0}^{m}\left|b_{k}\right||u|^{k}|B|^{2 k} y, y\right\rangle^{1 / 2}
\end{aligned}
$$

for any $x, y \in H$ and $m \in \mathbf{N}$.

Since the series whose partial sums are involved in the inequality (3.16) are convergent, then taking the limit over $m \rightarrow \infty$ in (3.16) we deduce the desired result (3.13).

COROLlaRY 2. Let $f(z)=\sum_{n=0}^{\infty} a_{n} z^{n}$ and be $g(z)=\sum_{n=0}^{\infty} b_{n} z^{n}$ be two functions defined by power series with real coefficients and both of them convergent on the open disk $D(0, R) \subset \mathbf{C}, R>0$. If $A$ and $B$ are two normal operators on the Hilbert space $H$ that 
satisfy condition (3.12) then we have

$$
\left\|g\left(\bar{u} B^{*}\right) f(z A)\right\|^{2} \leq f_{a}\left(|z|^{2}\right) g_{a}\left(|u|^{2}\right)\left\|f_{a}\left(|A|^{2}\right)\right\|\left\|g_{a}\left(|B|^{2}\right)\right\|
$$

and

$$
w\left(g\left(\bar{u} B^{*}\right) f(z A)\right) \leq \frac{1}{2} f_{a}\left(|z|^{2}\right) g_{a}\left(|u|^{2}\right)\left\|f_{a}\left(|A|^{2}\right)+g_{a}\left(|B|^{2}\right)\right\|
$$

\section{Some Examples}

As some natural examples that are useful for applications, we can point out that, if

$$
\begin{aligned}
& f(z)=\sum_{n=1}^{\infty} \frac{(-1)^{n}}{n} z^{n}=\ln \frac{1}{1+z}, \quad z \in D(0,1) ; \\
& g(z)=\sum_{n=0}^{\infty} \frac{(-1)^{n}}{(2 n) !} z^{2 n}=\cos z, \quad z \in \mathbf{C} ; \\
& h(z)=\sum_{n=0}^{\infty} \frac{(-1)^{n}}{(2 n+1) !} z^{2 n+1}=\sin z, \quad z \in \mathbf{C} ; \\
& l(z)=\sum_{n=0}^{\infty}(-1)^{n} z^{n}=\frac{1}{1+z}, \quad z \in D(0,1) ;
\end{aligned}
$$

then the corresponding functions constructed by the use of the absolute values of the coefficients are

$$
\begin{aligned}
& f_{a}(z)=\sum_{n=1}^{\infty} \frac{1}{n} z^{n}=\ln \frac{1}{1-z}, \quad z \in D(0,1) ; \\
& g_{a}(z)=\sum_{n=0}^{\infty} \frac{1}{(2 n) !} z^{2 n}=\cosh z, \quad z \in \mathbf{C} ; \\
& h_{A}(z)=\sum_{n=0}^{\infty} \frac{1}{(2 n+1) !} z^{2 n+1}=\sinh z, \quad z \in \mathbf{C} ; \\
& l_{A}(z)=\sum_{n=0}^{\infty} z^{n}=\frac{1}{1-z}, \quad z \in D(0,1) .
\end{aligned}
$$

Other important examples of functions as power series representations with nonnegative coefficients are:

$$
\exp (z)=\sum_{n=0}^{\infty} \frac{1}{n !} z^{n} \quad z \in \mathbf{C}
$$




$$
\begin{aligned}
\frac{1}{2} \ln \left(\frac{1+z}{1-z}\right) & =\sum_{n=1}^{\infty} \frac{1}{2 n-1} z^{2 n-1}, \quad z \in D(0,1) \\
\sin ^{-1}(z) & =\sum_{n=0}^{\infty} \frac{\Gamma\left(n+\frac{1}{2}\right)}{\sqrt{\pi}(2 n+1) n !} z^{2 n+1}, \quad z \in D(0,1) \\
\tanh ^{-1}(z) & =\sum_{n=1}^{\infty} \frac{1}{2 n-1} z^{2 n-1}, \quad z \in D(0,1) \\
{ }_{2} F_{1}(\alpha, \beta, \gamma, z) & =\sum_{n=0}^{\infty} \frac{\Gamma(n+\alpha) \Gamma(n+\beta) \Gamma(\gamma)}{n ! \Gamma(\alpha) \Gamma(\beta) \Gamma(n+\gamma)} z^{n}, \alpha, \beta, \gamma>0 \\
z & \in D(0,1)
\end{aligned}
$$

where $\Gamma$ is Gamma function.

On making use of Theorem 1, we can state some particular examples as follows:

EXAMPLE 1. a) If $A$ and $B$ are two bounded operators on the Hilbert space $H$ and for $p>1, \frac{1}{p}+\frac{1}{q}=1,\|A\|,\|B\|<1$, then

$$
\left\|\left(1_{H} \pm A B\right)^{-1}\right\| \leq \min \left\{S_{1}(p, q), S_{2}(p, q)\right\}
$$

where

$$
S_{1}(p, q):=\left(1-\|A\|^{p}\right)^{-1 / p}\left(1-\|B\|^{q}\right)^{-1 / q},
$$

and

$$
S_{2}(p, q):=\frac{1-\|A\|^{p-1}\|B\|^{q-1}}{\left(1-\|A\|^{p}\right)\left(1-\|B\|^{q}\right)} .
$$

We also have the following inequality for the logarithm

$$
\left\|\ln \left(1_{H} \pm A B\right)^{-1}\right\| \leq \min \left\{T_{1}(p, q), T_{2}(p, q)\right\}
$$

where

$$
T_{1}(p, q):=\left[\ln \left(1-\|A\|^{p}\right)^{-1}\right]^{1 / p}\left[\ln \left(1-\|B\|^{q}\right)^{-1}\right]^{1 / q}
$$

and

$$
T_{2}(p, q):=\frac{\left[\ln \left(1-\|A\|^{p}\right)^{-1}\right]\left[\ln \left(1-\|B\|^{q}\right)^{-1}\right]}{\ln \left(1-\|A\|^{p-1}\|B\|^{q-1}\right)^{-1}} .
$$
then

b) If $A$ and $B$ are two bounded operators on the Hilbert space $H$ and $p>1, \frac{1}{p}+\frac{1}{q}=1$,

$$
\|\exp (A B)\| \leq \min \left\{U_{1}(p, q), U_{2}(p, q)\right\}
$$


where

$$
U_{1}(p, q):=\exp \left(\frac{1}{p}\|A\|^{p}+\frac{1}{q}\|B\|^{q}\right)
$$

and

$$
U_{2}(p, q):=\exp \left(\|A\|^{p}+\|B\|^{q}-\|A\|^{p-1}\|B\|^{q-1}\right) .
$$

Theorem 2 provides the following results for unitarily invariant norm $|\| \cdot|||$ on a finite matrix algebra.

EXAMPLE 2. a) Let $\|\cdot\|$ be a unitarily invariant norm on a finite matrix algebra. If $\left\||A|^{p}\right\|\left|,\left\||B|^{q}\right\|\right|<1$, where $p>1, \frac{1}{p}+\frac{1}{q}=1$, then

$$
\left\|(I \pm A B)^{-1}\right\| \mid \leq \min \left\{V_{1}(p, q), V_{2}(p, q)\right\}
$$

where

$$
V_{1}(p, q):=\left(1-\left\|\left.|| A\right|^{p}\right\|\right)^{-1 / p}\left(1-\left\|\left.|| B\right|^{q}\right\|\right)^{-1 / q},
$$

and

$$
V_{2}(p, q):=\frac{1-\left\|\left.|| A\right|^{p}\right\|^{1 / q}\left\|\left.|| B\right|^{q}\right\|^{1 / p}}{\left(1-\left\|\left.|| A\right|^{p}\right\|\right)\left(1-\left\|\left.|| B\right|^{q}\right\|\right)}
$$

and

$$
\left|\| \ln (I \pm A B)^{-1}\right| \mid \leq \min \left\{W_{1}(p, q), W_{2}(p, q)\right\}
$$

where

$$
W_{1}(p, q):=\left[\ln \left(1-\left\|\left.|| A\right|^{p}\right\|\right)^{-1}\right]^{1 / p}\left[\ln \left(1-\left\|\left.|| B\right|^{q}\right\|\right)^{-1}\right]^{1 / q},
$$

and

$$
W_{2}(p, q):=\frac{\ln \left(1-\left\|\left.|| A\right|^{p}\right\|\right)^{-1} \ln \left(1-\left\|\left.|| B\right|^{q}\right\|\right)^{-1}}{\ln \left(1-\left.\left\||||A|^{p}\left|\left\|^{1 / q}\right\|\right||B|^{q}\right\|\right|^{1 / p}\right)^{-1}} .
$$

b) For any two matrices we have

$$
\|\exp (A B) \mid\| \leq \min \left\{Z_{1}(p, q), Z_{2}(p, q)\right\}
$$

where

$$
Z_{1}(p, q):=\exp \left(\frac{1}{p}\left\|\left.|| A\right|^{p}\right\|\left|+\frac{1}{q}\left\|\left.|| B\right|^{q}\right\|\right),\right.
$$

and

$$
Z_{2}(p, q):=\exp \left(\left\|\left.|| A\right|^{p}\right\|\left|+\left\|\left.|| B\right|^{q}\right\|\right|-\left.\left\|\left.|| A\right|^{p}\right\|\right|^{1 / q}\left\||B|^{q}\right\|^{1 / p}\right) .
$$


Employing the inequalities from Theorem 3 and Remark 3 we can state:

EXAmple 3. a) If $A$ and $B$ are two bounded operators on the Hilbert space $H$ and

$$
\|A\|,\|B\|<1,
$$

then

$$
\begin{aligned}
\left\|\left(1_{H} \pm A B\right)^{-1}\right\|^{2} & \leq\left(1-\|A\|^{1+\alpha}\|B\|^{1-\alpha}\right)^{-1}\left(1-\|A\|^{1-\alpha}\|B\|^{1+\alpha}\right)^{-1} \\
& \leq\left(1-\|A\|^{2}\right)^{-1}\left(1-\|B\|^{2}\right)^{-1}
\end{aligned}
$$

and

$$
\begin{aligned}
& \left\|\ln \left(1_{H} \pm A B\right)^{-1}\right\|^{2} \\
& \quad \leq \ln \left(1-\|A\|^{1+\alpha}\|B\|^{1-\alpha}\right)^{-1} \ln \left(1-\|A\|^{1-\alpha}\|B\|^{1+\alpha}\right)^{-1} \\
& \quad \leq \ln \left(1-\|A\|^{2}\right)^{-1} \ln \left(1-\|B\|^{2}\right)^{-1},
\end{aligned}
$$

where $\alpha \in[0,1]$.

b) For any bounded linear operators $A$ and $B$ we have the inequalities

$$
\begin{aligned}
\|\exp (A B)\|^{2} & \leq \exp \left(\|A\|^{1+\alpha}\|B\|^{1-\alpha}+\|A\|^{1-\alpha}\|B\|^{1+\alpha}\right) \\
& \leq \exp \left(\|A\|^{2}+\|B\|^{2}\right),
\end{aligned}
$$

where $\alpha \in[0,1]$, and

$$
\|\exp (z A B)\| \leq \exp \left(|z|\left(1+\|A\|^{2}+\|B\|^{2}-\|A\|-\|B\|\right)\right),
$$

where $z \in \mathbf{C}$.

Finally, by the use of the result in Theorem 5 we also have:

EXAMPLE 4. a) If $A$ and $B$ are two commuting normal operators on the Hilbert space $H$ with $\|A\|,\|B\|<1$ and $z \in D(0,1)$ then we have the inequalities

$$
\begin{aligned}
\left|\left\langle\left(1_{H} \pm z A B\right)^{-1} x, y\right\rangle\right|^{2} & \\
\leq & \left\langle\left(1_{H}-|z||A|^{2}\right)^{-1} x, x\right\rangle\left\langle\left(1_{H}-|z||B|^{2}\right)^{-1} y, y\right\rangle, \\
\left|\left\langle\ln \left(1_{H} \pm z A B\right)^{-1} x, y\right\rangle\right|^{2} & \leq\left\langle\ln \left(1_{H}-|z||A|^{2}\right)^{-1} x, x\right\rangle\left\langle\ln \left(1_{H}-|z||B|^{2}\right)^{-1} y, y\right\rangle,
\end{aligned}
$$

and

$$
\begin{aligned}
& \|\left\langle{ }_{2} F_{1}(\alpha, \beta, \gamma, z A B) x,\left.y\right|^{2}\right. \\
& \quad \leq\left\langle{ }_{2} F_{1}\left(\alpha, \beta, \gamma,|z||A|^{2}\right) x, x\right\rangle\left\langle{ }_{2} F_{1}\left(\alpha, \beta, \gamma,|z||B|^{2}\right) y, y\right\rangle
\end{aligned}
$$

where $\alpha, \beta, \gamma>0$, for any $x, y \in H$. 
b) If $A$ and $B$ are two commuting normal operators on the Hilbert space $H$ and $z \in \mathbf{C}$ then we have the inequalities

$$
\begin{aligned}
& |\langle\sin (z A B) x, y\rangle|^{2},|\langle\sinh (z A B) x, y\rangle|^{2} \\
& \quad \leq\left\langle\sinh \left(|z||A|^{2}\right) x, x\right\rangle\left\langle\sinh \left(|z||B|^{2}\right) y, y\right\rangle, \\
& \quad \leq\left\langle\cosh \left(|z||A|^{2}\right) x, x\right\rangle\left\langle\cosh \left(|z||B|^{2}\right) y, y\right\rangle
\end{aligned}
$$

and

$$
|\langle\exp (z A B) x, y\rangle|^{2} \leq\left\langle\exp \left(|z||A|^{2}\right) x, x\right\rangle\left\langle\exp \left(|z||B|^{2}\right) y, y\right\rangle
$$

for any $x, y \in H$.

ACKNOWLedgement. The authors would like to thank the anonymous referee for valuable comments that have been implemented in the final version of the paper.

\section{References}

[ 1 ] R. Bhatia, Matrix Analysis, Springer, 1997

[2] S. S. Dragomir, A survey on Cauchy-Bunyakovsky-Schwarz type discrete inequality, J. Ineq. Pure \& Appl. Math. 4 (2003), No. 3, Art. 63, pp. 142. [Online http://www.emis.de/journals/JIPAM/article301. html?sid=301]

[ 3 ] S. S. Dragomir, On some inequalities (Romanian), Caiete Metodico-Stiintifice, No. 13, 1984, pp. 20. Faculty of Mathematics, Timisoara University, Romania.

[ 4 ] S. S. Dragomir, Generalizations of Furuta's inequality, Linear and Multilinear Algebra 61 (2013), no. 5, 617-625; Preprint RGMIA Res. Rep. Coll. 15 (2011), Art. 15, pp. 10. [Online http://rgmia.org/v15.php].

[ 5 ] S. S. DRAGomir, Some inequalities of Furuta's type for functions of operators defined by power series, RGMIA Res. Rep. Coll. 15 (2011), Art. 17, pp. 12. [Online http://rgmia.org/v15.php].

[ 6 ] S. S. DRAGOMIR and J. SÁNDOR, Some generalisations of Cauchy-Buniakowski-Schwartz's inequality (Romanian), Gaz. Mat. Metod. (Bucharest) 11 (1990), 104-109.

[ 7 ] T. Furuta, An extension of the Heinz-Kato theorem, Proc. Amer. Math. Soc. 120 (1994), no. 3, 785-787.

[ 8 ] M. UchiYama, Further extension of the Heinz-Kato-Furuta inequality, Proc. Amer. Math. Soc. 127 (1999), no. 10, 2899-2904.

Present Addresses:

SEVER SiLVESTRU Dragomir

Mathematics, College of ENGineERING \& SCIENCE,

VICTORIA UNIVERSITY,

PO Box 14428, Melbourne City, MC 8001, Australia.

SChool of Computational \& ApPlied Mathematics,

UNIVERSITY OF THE WITWATERSRAND,

Private Bag 3, JohanNesburg 2050, South Africa.

e-mail: sever.dragomir@vu.edu.au

URL: http://rgmia.org/dragomir 
Mitsuru UChiYAMA

DEPARTMENT OF MATHEMATICS,

SHIMANE UNIVERSITY,

Matsue City, Shimane, JAPAN.

e-mail: uchiyama@riko.shimane-u.ac.jp 